03

\title{
Динамика капель, подброшенных над испаряющейся поверхностью
} воды

\author{
(ㄱ Д.Н. Габышев, ${ }^{1}$ Д.Н. Медведев, ${ }^{1}$ К.В. Мисиюк ${ }^{2}$ \\ ${ }^{1}$ Тюменский государственный университет, Институт экологической и сельскохозяйственной биологии (X-BIO), \\ 625003 Тюмень, Россия \\ ${ }^{2}$ Университет Отаго, Департамент физики, \\ 9016 Данидин, Новая Зеландия \\ e-mail: gabyshev-dmitrij@rambler.ru
}

Поступило в Редакцию 3 февраля 2021 г.

В окончательной редакции 13 апреля 2021 г.

Принято к публикации 16 апреля 2021 г.

Проанализированы данные баллистического эксперимента, в котором интенсивная капиллярная волна подбрасывает микрокапли, сформированные и левитирующие над нагретым участком воды за счет восходящего конвективного паровоздушного потока. Оценивается сопротивление движению капли со стороны этого потока. С использованием различных теоретических приближений оцениваются параметры потока (скорость на разных высотах, быстрота убывания скорости). Определен максимальный размер капель, могущих левитировать свободно. Показана невозможность стационарной левитации капель в линейно неоднородном потоке.

Ключевые слова: межфазная поверхность вода-воздух, капиллярная волна, капельный кластер, закон Стокса, прыжок капли, гидродинамическая интерференция.

DOI: 10.21883/JTF.2021.09.51211.25-21

\section{Введение}

В 1922 г. Торахико Терада обратил внимание на физические явления, сопровождающие существование плоской белой туманной дымки над чашкой горячего чая [1]. В 1971 г. В. Шефер направил оптический микроскоп на нее и обнаружил зависшие микроскопические капельки воды. Они были не такими легкими, чтобы улететь прочь, но и не такими тяжелыми, чтобы упасть вниз: восходящий паровоздушный поток компенсировал их силу тяжести [2]. При этом капли были разбросаны над поверхностью воды весьма хаотично [3]. Исследователи научились собирать подобные капельки в небольшие маломобильные и легко наблюдаемые упорядоченные группы к 2004 г. [4]. Для этого применялся локальный нагрев воды в кювете с помощью расположенного снизу тонкого нагревателя диаметром порядка $1 \mathrm{~mm}$, а слой испаряемой воды брался толщиной менее $1 \mathrm{~mm}$. Первое условие было необходимо для пространственной локализации массива левитирующих капель, а второе для подавления термокапиллярной конвекции, вызывающей хаотическое движение водной поверхности слоя. Упорядоченные левитирующие массивы были названы капельными кластерами. Типичный диаметр капель в кластерах находится в диапазоне между 10 и $100 \mu \mathrm{m}$. Кластеры исследовались не только как самостоятельный причудливый феномен, но они выступили также и как инструмент для решения фундаментальных исследовательских задач. Например, некоторый вклад был сделан в микрометеорологию: был изучен конденсационный рост микрокапель в электрическом поле [5-8], а также было положено начало изучению электростатической коалесценции [7-9]. Оба процесса происходят внутри атмосферных облаков. Также было исследовано колебательно-подобное движение капельных кластеров [10], которое присуще жидким гидрометеорам при их выпадении на землю с терминальной скоростью. Недавние эксперименты продемонстрировали принципиальную возможность лабораторного изучения биоаэрозолей, засеянных одноклеточными водорослями и бактериями [11]. Наметились приложения в области вирусологии [12]. В мире получили распространение несколько аналогов используемой лабораторной установки $[1,13,14]$. Недавно вышедший обзор о левитации капель показал, что технология капельного кластера далеко не исчерпала своих возможностей и остается актуальной [15].

С самого начала исследований [16] осуществлялись поиски механизмов, ответственных за существование капельных кластеров. Постепенно стало понятно, что определяющую роль играет паровоздушный поток, поддерживающий левитацию капель $[17,18]$. Этот поток является частью сложного конвективного факела [19], подтекающего со всех сторон и вздымающегося над точкой локального нагрева $[18,20]$. Данный конвективный факел характеризуется высоким температурным градиентом по вертикали [21]. Приток воздуха по горизонтали извне в радиальном направлении от периферии к центру области нагрева происходит из-за наличия существенного градиента температур вдоль по поверхности подстилающего слоя воды (по причине того же локального характера нагрева) [22-24]. 
Аэродинамический механизм левитации капель в вертикальном потоке конвективного факела является общепризнанным $[17,18,25,26]$. Однако новейшие наблюдения с высокочастотной видеосъемкой в работе [27] выявили, что простой силы Стокса недостаточно для левитации наиболее тяжелых наблюдаемых капель. Целью настоящей работы, во-первых, является иллюстрация наблюдаемой неувязки и попытка ее объяснения. Вовторых, в рамках различных приближений попутно оцениваются аэродинамические параметры паровоздушного потока (вертикальная компонента скорости и ее градиент) на основе данных из [27]. Также рассматривается возможность стационарной левитации свободной капли в восходящем влажном потоке.

\section{1. Описание экспериментальной процедуры}

Экспериментальная установка подробно описана в работе [27], поэтому здесь мы перечислим лишь ее ключевые характеристики. Слой воды толщиной $200 \mu \mathrm{m}$ распределен по плоской ситалловой подложке, которая подогревается снизу инфракрасным лазером KLM-H808600-5 с постоянной мощностью. В результате температура поверхности воды в центре подложки поддерживается на уровне $80^{\circ} \mathrm{C}$. Наблюдение ведется через стереомикроскоп МБС-10 со скоростной видеокамерой Photron FASTCAM SA1.1 (частота записи 20000 кадров в секунду).

Во влажном воздухе, восходящем над областью нагрева, случайным образом возникают капли чистой воды на естественных центрах конденсации. Ряд капель имеет такой вес, что он уравновешивается лобовым сопротивлением от восходящего потока. Постепенно такие левитирующие капли выстраиваются в кластер, упорядоченный в силу естественных законов [24]. Капли кластера непрерывно растут из-за конденсации влаги из окружающего их воздуха. В некоторый момент вырастает капля настолько тяжелая, что она не может более левитировать. Она касается подстилающей поверхности воды и сливается с нею под действием каплиллярных сил и, в гораздо меньшей мере, гравитации. В этот момент на межфазной поверхности генерируются быстрые капиллярные волны, которые вызывают цепную реакцию коалесценции соседних капель. Данный механизм был подробно изучен в серии статей [7-9,28-30].

Из-за лавинообразной коалесценции капель в центре кластера капиллярные волны интерферируют, так что какая-то капля может оказаться в области сходящихся волн. Капля оказывается в углублении, которое мгновенно сужается, и капля подбрасывается вертикально вверх на высоту до нескольких своих диаметров. При этом капиллярная волна успевает рассеяться значительно быстрее, чем длится свободный полет капли. Сложившаяся ситуация сводится к простой баллистической задаче для тела, движущегося в поле земного тяготения при наличии вертикального потока среды. В настоящее время подобные прыжки капель интересуют широкий круг исследователей, изучающих физические системы различного типа [31-34].

В работе [27] наблюдался выброс сразу двух капель диаметром $A 71 \pm 0.6 \mu \mathrm{m}$ и $B 86 \pm 0.6 \mu \mathrm{m}$. Капля $A$ была подброшена на максимальную высоту $417 \pm 0.6 \mu \mathrm{m}$, отсчитываемую от центра капли (около шести ее диаметров). Капля В была подброшена на высоту $131 \pm 0.6 \mu \mathrm{m}$ (полтора ее диаметра). Длительность свободного полета составляла около $25.5 \pm 0.025 \mathrm{~ms}$ для легкой капли и $12.0 \pm 0.025 \mathrm{~ms}$ для тяжелой капли. По окончании полета обе капли упали на поверхность подстилающего слоя воды и слились с нею.

Скорость капли $A$ в начальный и конечный моменты наблюдения была равной $6.3 \pm 0.1$ и $5.3 \pm 0.1 \mathrm{~cm} / \mathrm{s}$ coответственно (кинетическая энергия, а с нею и скорость уменьшилась из-за работы сил трения, действующих вдоль траектории капли). Для капли В скорость в начале и конце составляла $2.7 \pm 0.1$ и $2.8 \pm 0.1 \mathrm{~cm} / \mathrm{s}$. Тот факт, что конечная скорость капли $B$ оказалась больше начальной, не противоречит закону сохранения механической энергии, потому что в момент начала наблюдения капля находилась на большей высоте $(56 \mu \mathrm{m})$, чем в конце наблюдения $(43 \mu \mathrm{m})$, и работа силы тяжести для капли смогла перекрыть работу сопротивления среды вдоль траектории. Для капли же $A$ разница между начальной $(52 \mu \mathrm{m})$ и конечной высотой $(36 \mu \mathrm{m})$ оказалась недостаточной, чтобы компенсировать работу сил трения.

Поскольку скорость капиллярных волн составляет около $2 \mathrm{~m} / \mathrm{s}$ [29], а диаметр капельного кластера в эксперименте около $1 \mathrm{~mm}$, чтобы капиллярной волне пробежать от первой коалесцирующей капли до капель на самом дальнем краю кластера, вызвать их коалесценцию и, отразившись, пробежать исходную точку в обратном направлении, требуется не более $0.001 \mathrm{~s}$. Очевидно, что примерно такое же время нужно, чтобы движение воздуха над слоем воды вновь пришло в стационарное состояние. То есть нестационарный режим охватывает период времени около $0.002 \mathrm{~s}$. Капли $A$ и $B$ подбрасывались, конечно, не в самый момент начала коллапса кластера, а только когда сходящиеся капиллярные волны создали благоприятную ситуацию, что имеет место лишь во второй половине процесса коллапса кластера. Траектории капель стали отслеживаться не сразу в момент броска, а только когда полностью исчезли соседние капли, мешавшие наблюдению, и стало четко видно границы капель $A$ и $B$. Таким образом, период нестационарного режима газовой среды, в которой двигались наблюдаемые капли, составлял не более $0.001 \mathrm{~s}$ или около $4 \%$ от времени движения легкой капли $A$ и $8 \%$ от времени движения тяжелой капли $B$. Безусловно, об этом следует помнить, так как это вносит некоторую неточность в последующие оценки. 


\section{2. Физическая модель}

Рассмотрим движение капли, подброшенной капиллярной волной. Пусть начальная высота левитации у капли массой $m$ и радиусом $R$ равна $H$, и капля подбрасывается строго вверх. Даже если вращение капли присутствует, мы пренебрежем силой Магнуса из-за ее предельной малости (система, в которой вращение капли потенциально могло бы привести к каким-либо внешним эффектам, изучается, например, в [35]). За счет капиллярных сил поверхность капли все время остается сферической. Второй закон Ньютона для нее (рис. 1):

$$
\begin{gathered}
m \ddot{\mathbf{y}}=m \mathbf{g}+\mathbf{F}_{\mathbf{b}}+\mathbf{F}_{\mathbf{d}}, \\
m \ddot{y}=-m g+F_{b}-F_{d} .
\end{gathered}
$$

Сила Архимеда $\mathbf{F}_{\mathbf{b}}$ не превышает тысячную долю от веса капли $m g$, поэтому пренебрегаем ею и выражаем силу $F_{d}$ сопротивления со стороны воздушной среды, окружающей ускоренно движущуюся каплю:

$$
\frac{F_{d}}{m g}=-1-\frac{\ddot{y}}{g} \text {. }
$$

Вычисление силы (3) корректно, если изменение $F_{d}$ на расстоянии диаметра капли много меньше силы тяжести: $\left.\frac{F_{d}(y)}{m g}\right|_{y-R} ^{y+R} \ll 1$, и это условие соблюдается по всей траектории движения капли (рис. 2). Следовательно, если из эксперимента известны все положения капли $y(t)$ (соответственно также скорости $\dot{y}(t)=d y / d t$ и ускорения $\left.\ddot{y}(t)=d^{2} y / d t^{2}\right)$, то можно понять закон, по которому меняется сила $F_{d}$. Таким образом, в данном случае капля служит в определенном смысле индикаторной частицей, позволяющей понять кое-что об обстановке вокруг нее. Рис. 2 показывает, что во время полета расчетная сила

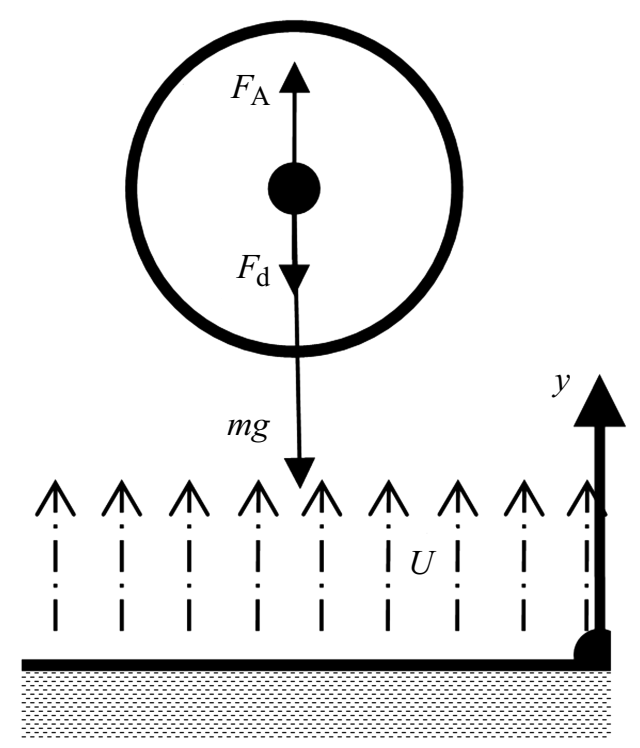

Рис. 1. Микрокапля с указанием действующих на нее сил в произвольный момент времени.

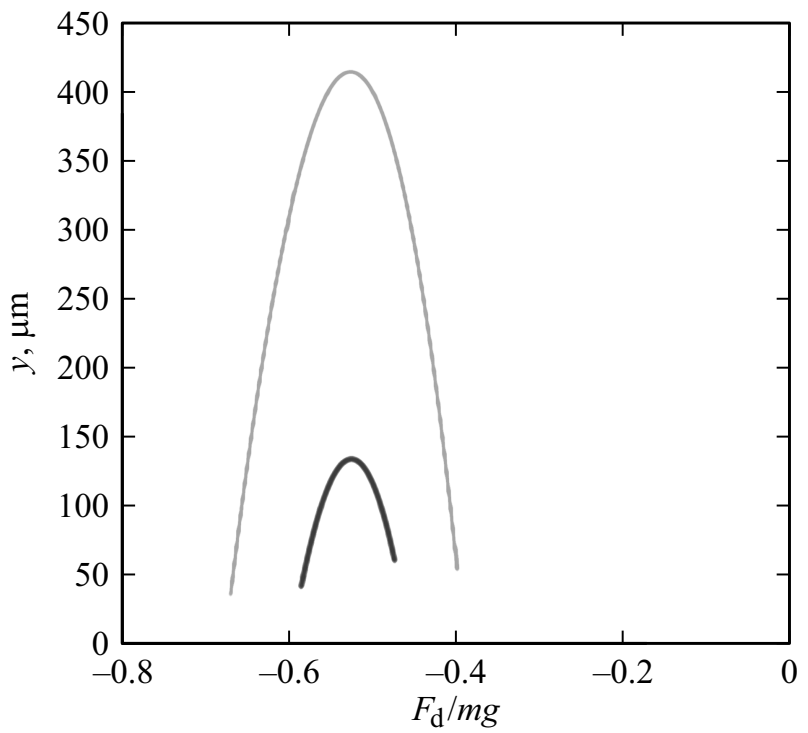

Рис. 2. Сила сопротивления, действующая на капли в процессе их движения: серая кривая - на легкую каплю $(A)$, черная кривая - на тяжелую каплю $(B)$.

$F_{d}$ постепенно растет по модулю (началу движения капли соответствует правый край изображенных кривых, a окончанию полета - левый край кривых). Причиной этого является то, что сила сопротивления среды пропорциональна модулю разности скорости потока $U$ и скорости капли $\dot{y}$. Этот модуль увеличивается, потому что происходит торможение капли в поле земной тяжести, и скорость $\dot{y}$ убывает. Сила $F_{d}$ отрицательна по знаку. Это значит, что ее направление противоположно выбранному на рис. 1. Капля в полете не смогла обогнать поток, так, чтобы сила $F_{d}$ стала бы положительной и была направлена вниз.

\section{3. Аналитические приближения}

Коэффициент сопротивления формы для шара с числом Рейнольдса Re, близким к единице, каковым нередко проявляет себя капля в кластере, вычисляется обыкновенно по формуле Озеена [36,37]

$$
C_{d}=\frac{24}{\operatorname{Re}}\left(1+\frac{3}{16} \operatorname{Re}\right)
$$

а соответствующее сопротивление среды имеет вид

$$
F_{O s}=6 \pi R \mu(\dot{y}-U)+\frac{9}{4} \pi \rho_{m} R^{2}|\dot{y}-U|(\dot{y}-U),
$$

где $\mu-$ динамическая вязкость влажного воздуха, $\rho_{m}-$ его плотность, $(\dot{y}-U)-$ скорость капли $\dot{y}$ относительно среды, движущейся со скоростью $U$, а $R-$ радиус капли.

Скорость $U$ играет здесь роль эффективной скорости среды. Необходимость ввести такую в некотором смысле усредненную скорость возникает потому, что поток 
меняет свою скорость, поднимаясь вверх. Вместе с тем точный закон для скорости потока исследователям пока не известен, а предварительные численные расчеты указывают на признаки его нелинейности $[38,39]$. Поэтому любые оценки параметров паровоздушного потока из реальных экспериментов представляют интерес. В связи с этим значительно проще оперировать некоторым усредненным значением $U$, а применение его будет тем адекватнее, чем слабее градиент скорости потока.

Таким образом, сила сопротивления приближенно записывается нами в виде

$$
\frac{F_{O s}}{m g}=\frac{9 \mu}{2 R^{2} \rho_{w} g}(\dot{y}-U)+\frac{27}{16} \frac{\rho_{m}}{R \rho_{w} g} \frac{(\dot{y}-U)^{3}}{|\dot{y}-U|},
$$

где $\rho_{w}-$ плотность воды. Поиск скорости $U$ состоит в нахождении такого ее значения, которое максимально сближает кривые (3) с (6). Это делается методом наименьших квадратов. Очевидно, что значение эффективной скорости $U$ будет несколько отличаться для легкой и тяжелой капель. Причиной тому служит то, что легкая капля заметает собою более обширную область, в которой скорость потока в среднем ниже, чем в непосредственной близости к поверхности воды, где сосредоточена траектория тяжелой капли. Стоит также отметить, что для каждой капли ее значение $U$ является завышенным по сравнению с истинным значением в наивысшей точке траектории. Причина состоит в том, что на среднее значение оказывает влияние множество точек начального и конечного участка пути, пролегающих вблизи поверхности воды, где скорость потока выше, чем в области, отдаленной от воды. Тем не менее капля проходит свой начальный и конечный отрезок достаточно быстро и дольше задерживается именно вблизи своей наивысшей точки (так как там скорость капли близка к нулю). Это позволяет надеяться на достаточно малый вклад в $U$ от нижних точек траектории.

В работе [27] внимание было уделено сближению аналитических траекторий капель с экспериментальными с помощью метода наименьших квадратов. Идея использовать траектории капель для расчета скорости газа над сухой нагретой поверхностью была упомянута ранее в [40], а возможный механизм левитации капель при таких условиях обсуждался в [41]. В настоящей работе мы определяем параметр $U$ с целью обеспечения расчетного сближения экспериментальных и аналитических сил (3) и (6), а не траекторий капель.

\section{4. Расчет}

Плотность паровоздушной смеси (воздух со 100\% влажностью при $80^{\circ} \mathrm{C}$ ) составляет $\rho_{m}=0.828 \mathrm{~kg} / \mathrm{m}^{3}$, плотность воды $\rho_{w}=971.8 \mathrm{~kg} / \mathrm{m}^{3}$, а динамическая вязкость $\mu=1.63 \cdot 10^{-5} \mathrm{~Pa} \cdot \mathrm{s}$ [42]. Ускорение свободного падения принимаем равным $g=9.81 \mathrm{~N} / \mathrm{kg}$. Методом наименьших квадратов отыскиваем значения $U$, которые обеспечивают максимальное совпадение (3) и (6):

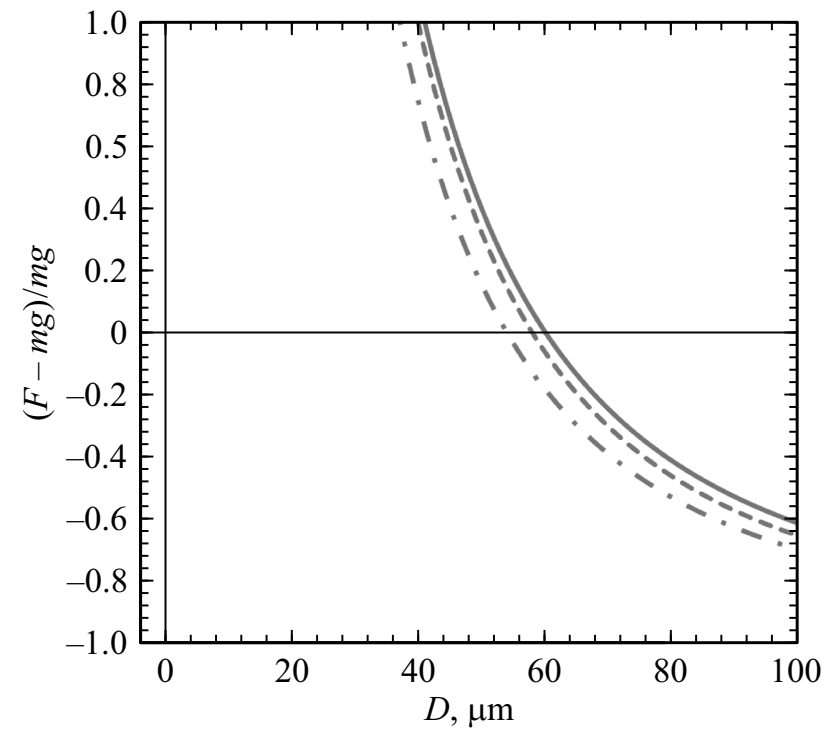

Рис. 3. Соотношение аэродинамической силы и веса капли в приближении Озеена (сплошная линия), приближении Стокса (штриховая линия) и из работы [27] (штрихпунктирная линия).

$U=8.1 \pm 0.1 \mathrm{~cm} / \mathrm{s}$ по легкой капле $A$ и $11.7 \pm 0.1 \mathrm{~cm} / \mathrm{s}$ по тяжелой капле $B$. Расстояние между наивысшими точками траекторий капель составляет $286 \pm 1.2 \mu \mathrm{m}$. Отсюда следует наличие вертикального градиента на уровне приблизительно $k=125.8 \pm 8.8(\mathrm{~m} / \mathrm{s}) / \mathrm{m}$.

Числа Рейнольдса Re, оцененные по максимальной скорости $U$, равны 0.293 для легкой капли $A$ и 0.352 для тяжелой капли $B$ при их левитации на постоянной высоте (до момента коллапса кластера). В таких условиях приближение Озеена (5) до 10\% точнее, чем приближение Стокса. Кроме того, максимальная скорость относительно газовой среды была достигнута легкой каплей $A$ в конце своего движения, непосредственно перед слиянием со слоем воды. В тот момент капля двигалась с собственной скоростью $5.3 \mathrm{~cm} / \mathrm{s}$ навстречу среде, набегающей со скоростью $11.7 \mathrm{~cm} / \mathrm{s}$ (оцененной выше). То есть максимальная скорость капли относительно среды составляла около $17.0 \mathrm{~cm} / \mathrm{s}$. Это соответствует значению числа Рейнольдса 0.613. Для тяжелой капли $B$ собственная скорость была равной $2.8 \mathrm{~cm} / \mathrm{s}$ в конечный момент падения, поэтому при движении навстречу паровоздушному потоку число Рейнольдса капли достигало 0.523. И в одном, и в другом случае это означает ламинарное обтекание капли на протяжении всей траектории.

Оценим максимальный размер капель, способных левитировать свободно. На рис. 3 приведена зависимость отношения $(F-m g) / m g$ от диаметра капли. В качестве силы $F$ подставляется приближение Озеена из (5) при $\dot{y}=0$, а также приближение Стокса, возникающее из (3) при пренебрежении квадратичным членом. Из рис. 3 следует, что максимальный диаметр капли, способной левитировать свободно, равен $61 \mu \mathrm{m}$, если опираться 
на приближение Озеена $F_{O s}$. При этом $F_{O s}$ составляет $76.6 \%$ от веса капли $A$ и $53.0 \%$ от веса капли $B$, т.е. в рамках приближения Озеена экспериментальный факт продолжительной левитации столь крупных капель не поддается объяснению. Данное противоречие впервые было обнаруженно в [27].

\section{5. К вопросу о стационарной левитации кластера}

Дополнительно проверим возможность стационарной левитации капли в процессе ее конденсационного роста при оцененном градиенте $k$. Известно [18], что небольшие капли, проникающие в кластер, могут левитировать на высоте до нескольких своих диаметров (при этом обтекание капель восходящим потоком препятствует их столкновению друг с другом). Можно предположить, что на этом этапе влияние поверхности подстилающего слоя воды минимально, из-за чего капля левитирует свободно. Для оценок достаточно рассмотреть только стоксовский режим левитации $[17,18,25,26]$.

Конденсационный рост капель кластера подчиняется закономерности, известной как закон $D^{2}$ (диаметр капли в квадрате) [25], которая выражается постоянной скоростью роста площади $S$ поверхности капли за единицу времени $\dot{S}=8 \pi \mathscr{D} \xi$ (здесь $\mathscr{D}-$ коэффициент диффузии водяного пара в воздухе, $\xi-$ пересыщение [43]). При этом для радиуса капли характерна корневая зависимость:

$$
R=\sqrt{R_{0}^{2}+B\left(t-t_{0}\right)},
$$

где $R_{0}$ - радиус в начальный момент наблюдения $t_{0}$. Константа $B$ равна $2 \mathscr{D} \xi$ или, что то же самое, величине $\dot{S} / 4 \pi$. Закон для роста массы капли тривиален:

$$
m=m_{0}+\frac{4 \pi \rho_{w}}{3}\left(R^{3}-R_{0}^{3}\right)
$$

где $m_{0}-$ начальная масса капли. Допустим, что в области свободной левитации кластера скорость потока линейно зависит от текущей высоты $y$ :

$$
U=U_{0}-k(y-H)
$$

где $U_{0}-$ скорость потока на равновесной высоте левитации $H$, при этом режим квазистационарный, т.е. в каждый момент времени вес капли можно считать уравновешенным силой Стокса:

$$
m g=6 \pi R \mu U
$$

Из (10) с учетом (7)-(9) высота левитации капли изменяется по закону

$$
y=H+\frac{U_{0}}{k}-\frac{g}{6 \pi R \mu k}\left[m_{0}+\frac{4 \pi \rho_{w}}{3}\left(R^{3}-R_{0}^{3}\right)\right] .
$$

Соответственно можно определить скорость проседания капли в линейно неоднородном паровоздушном течении (памятуя, что $R$ зависит от $t$ ):

$$
\dot{y}=-\frac{\dot{S} \rho_{w} g}{18 \pi \mu k} .
$$

Отсюда видим, что величина $\dot{y}$ постоянна, и какой бы ни был размер капли, она все равно не обращается в нуль. Подставив типичное значение скорости $\dot{S}=300 \mu \mathrm{m}^{2} / \mathrm{s}$ [25] и вычисленное значение градиента $k=125.8(\mathrm{~m} / \mathrm{s}) / \mathrm{m}$, получаем значение скорости $\dot{y}=-24.7 \mu \mathrm{m} / \mathrm{s}$. При такой скорости капля должна коснуться поверхности и слиться со слоем воды через время

$$
t=H / \dot{y}=3.65 \mathrm{~s} .
$$

Однако характерное время левитации кластера измеряется минутами. Налицо противоречие: непрерывно растущая капля не должна находиться в покое долгое время.

\section{6. Обсуждение}

Таким образом, два вышеуказанных факта говорят против свободной аэродинамической левитации наиболее тяжелых наблюдаемых капель: недостаточность подъемной силы (рис. 3) и долгая левитация капель в течение минут, которая, казалось бы, невозможна при их интенсивном росте (13). Эти противоречия можно объяснить тем, что гигантские капли в действительности не левитируют, а как бы лежат на поверхности воды благодаря тонкому промежуточному слою воздуха, зажатому между каплей и поверхностью воды, словно шайба в аэрохоккее [44-46]. При этом масса капель в подобных экспериментах остается все еще достаточно малой для того, чтобы под ними образовывался вогнутый капиллярный мениск. В эксперименте [27] это проявлялось в том, что зазор между каплей и водой не удавалось измерить из-за оптических ограничений микроскопа, так как он составлял не более $0.6 \mu \mathrm{m}$ (погрешность измерения). Формирующиеся при этом вихри и подтекание воздуха в область под каплей были зафиксированы с помощью трассерных частиц на поверхности воды [47]. Таким образом, вблизи поверхности воды преобладает механизм левитации, отличный от простой аэродинамической свободной левитации.

В [48] была выведена приближенная формула для силы сопротивления среды, которая учитывает воздушную подушку:

$$
F_{\mathrm{Sh}}=-6 \pi R \mu U\left(1+\frac{R}{H-R}\right) .
$$

Формула (14) качественно воспроизводит тот факт, что сила оказывается тем больше, чем меньше зазор под каплей. Однако она имеет то неудобство, что рост происходит слишком резко, и к тому же при нулевом 
зазоре $(H-R)$ сила обращается в бесконечность, хотя в реальных экспериментах наблюдается коалесценция. Поэтому в $[49,50]$ формула (14) частично корректировалась поправочными коэффициентами, рассчитанными по ранее опубликованным экспериментальным зависимостям $H$ от $R$ [18].

Во всех предыдущих случаях физика процесса оставалась за рамками выкладок исследований. Однако, повидимому, эффект воздушной подушки, когда зазор между каплей и поверхностью воды мал, включает в себя гидродинамическую интерференцию сферической капли с поверхностью воды. Данное явление увеличивает силу сопротивления и тем самым затрудняет прямой контакт при сближении. Говардом Бреннером в работах $[51,52]$ были получены выражения для силы сопротивления, оказываемой однородным и изотропным несжимаемым флюидом на твердую сферическую частицу, равномерно движущуюся со скоростью $U$ по направлению к свободной плоской поверхности или от нее:

$$
F_{\mathrm{Br}}=-6 \pi R \mu U \beta
$$

где $\beta-$ поправка к закону Стокса, задаваемая уравнением:

$$
\begin{aligned}
\beta & =\frac{4}{3} \sinh \alpha \sum_{n=1}^{\infty} \frac{n(n+1)}{(2 n-1)(2 n+3)} \\
\times & {\left[\frac{4 \cosh ^{2}\left(n+\frac{1}{2}\right) \alpha+(2 n+1)^{2} \sinh ^{2} \alpha}{2 \sinh (2 n+1) \alpha-(2 n+1) \sinh 2 \alpha}-1\right], }
\end{aligned}
$$

где, в свою очередь, $\alpha=\operatorname{acosh} \frac{H}{R}$.

В присутствии движущейся среды выражение (15) вместо $-U$ должно иметь множитель $(\dot{y}-U)$. Применив далее процедуру, описанную в разд. 3 и исключив при необходимости точку расходимости выражения (15) в момент коалесценции $\alpha=0$, можно показать, что метод наименьших квадратов дает $U=4.3 \pm 0.1 \mathrm{~cm} / \mathrm{s}$ по легкой капле и $5.7 \pm 0.1 \mathrm{~cm} / \mathrm{s}$ по тяжелой капле. Оба значения оказываются в два раза меньше, чем величины, полученные нами в разд. 4 по приближению Озеена. Вертикальный градиент скорости потока между наивысшими точками траекторий капель составляет приблизительно $k=48.9 \pm 8.8(\mathrm{~m} / \mathrm{s}) / \mathrm{m}$, что уже в два с половиной раза меньше величины, полученной в разд. 4. Он должен разниться по высоте, но, судя по всему, его порядок одинаков во всей области локализации траекторий капель. Значит, непосредственно на поверхности воды паровоздушный поток поднимается со скоростью около $7.1 \mathrm{~cm} / \mathrm{s}$. Однако при данном значении $U$ сила (15) дает многократно большее значение, чем необходимо для равновесия капель $A$ и $B$ до начала коллапаса кластера. Или, например, известно, что капля диаметром $40 \mu \mathrm{m}$ при той же температуре левитирует на высоте $H=47 \mu \mathrm{m} \mathrm{[18],} \mathrm{но} \mathrm{даже} \mathrm{для} \mathrm{нее} \mathrm{приближение} \mathrm{Брен-}$ нера (15) дает значение пусть не в разы, но на 95\% превышающее вес капли.
Поскольку приближения Стокса-Озеена недостаточно для объяснения левитации, а приближение Бреннера, наоборот, дает избыток, следующая картина представляется более вероятной. Гидродинамическая интерференция позволяет полностью компенсировать недостаток силы Стокса-Озеена, однако сам механизм гидродинамической интерференции проявляется не полностью, потому что имеются существенные отличия реальной ситуации от теоретической задачи, решенной Г. Бреннером [51]. Среди отличий можно указать, что газовая среда является сжимаемой молекулярной (а не сплошной) фазой. На границе вода-воздух происходит интенсивный фазовый переход, и капля сама постоянно растет. Паровоздушная среда движется от поверхности воды, чего нет в теоретическом приближении. Расположение линий тока среды в этом случае отлично от случая, когда среда была неподвижна [52]. Кроме того, реальная капля окружена слоем Кнудсена и пограничным слоем. Все эти факторы могут сильно „смазывать“ идеальную теоретическую картину. В связи со всем этим можно заключить, что имеет место неполная гидродинамическая интерференция капли с поверхностью воды.

\section{Заключение}

Левитация является онтологической характеристикой капельного кластера [4]. В ходе исследования были проанализированы данные баллистического эксперимента, в котором микрокапли воды, левитирующие над локально нагретым участком воды, были подброшены вертикально вверх интенсивной капиллярной волной [27].

Ранее считалось, что капли, из которых состоит капельный кластер, левитируют благодаря тому, что восходящий поток сопротивляется гравитации, стремящейся опустить капли вниз. Это полностью справедливо только для сравнительно малых капель. Реальная картина сложна, и чистая аэродинамическая левитация при температуре $80^{\circ} \mathrm{C}$ возможна только для капель диаметром не более $61 \mu \mathrm{m}$. Все капли, диаметр которых оказывается больше, требуют для поддержания своей левитации наличия под собой тонкого промежуточного слоя воздуха, предотвращающего коалесценцию [4446]. Кроме того, недостаток подъемной силы, по всей видимости, компенсируется имеющей место неполной гидродинамической интерференцией капли со свободной поверхностью воды. Численные оценки тех или иных величин зависят от заложенной теоретической модели. Похоже, что истинные значения лежат гдето посередине между теми, что получены при полном отсутствии гидродинамической интерференции, и теми, что получены при ее полноценном действии. Оценки могут быть скорректированы в будущем при проведении более точных исследований. Однако уже теперь был надежно установлен факт, что скорость паровоздушного потока, поддерживающего левитацию капель, убывает с высотой независимо от модели. В целом приходится 
признать, что картина левитации капельного кластера более сложна, чем представлялось недавно, и требуются дальнейшие исследования для понимания этого феномена.

\section{Финансирование работы}

Исследование было поддержано Советом по грантам президента РФ (грант № МК-819.2020.2) и частично Министерством науки и высшего образования РФ (проект № AAAA-A20-120051490005-9)

\section{Благодарности}

Д.Н. Габышев благодарит А.А. Федорца (Тюмень), а также профессоров И.В. Марчука и О.А. Кабова (Новосибирск) за предоставленные исходные данные о траекториях капель.

\section{Конфликт интересов}

Авторы заявляют, что у них нет конфликта интересов.

\section{Список литературы}

[1] T. Umeki, M. Ohata, H. Nakanishi, M. Ichikawa. Sci. Rep., 5, 8046 (2015). DOI: $10.1038 /$ srep08046

[2] V.J. Schaefer. Am. Sci., 59 (5), 534 (1971).

[3] F. Ienna, H. Yoo, G.H. Pollack. Soft Matter, 8, 11850 (2012). DOI: $10.1039 / \mathrm{c} 2 \mathrm{sm} 26497 \mathrm{~h}$

[4] А.А. Федорец. Письма в ЖЭТФ, 79 (8), 457 (2004). [A.A. Fedorets. JETP Lett., 79 (8), 372 (2004). DOI: $10.1134 / 1.1772434]$

[5] D.N. Gabyshev, A.A. Fedorets, O. Klemm. Aerosol Sci. Technol., 54, 1556 (2020). DOI: $10.1080 / 02786826.2020 .1804522$

[6] D.N. Gabyshev, A.A. Fedorets, N.E. Aktaev, O. Klemm, S.N. Andreev. J. Aerosol Sci., 135, 103 (2019). DOI: $10.1016 /$ j.jaerosci.2019.06.002

[7] A.A. Fedorets, L.A. Dombrovsky, E. Bormashenko, M. Nosonovsky. Int. J. Heat Mass Transf., 133, 712 (2019). DOI: 10.1016/j.jheatmasstransfer.2018.12.160

[8] A.A. Fedorets, L.A. Dombrovsky, E. Bormashenko, D.N. Gabyshev, M. Nosonovsky. Conf.: 14th Int. Conf. on Heat Transfer, Fluid Mech. and Thermodyn. (HEFAT-2019) at: Wicklow, Ireland. DOI: 10.13140/RG.2.2.26140.54400

[9] A.A. Fedorets, L.A. Dombrovsky, D.N. Gabyshev, E. Bormashenko, M. Nosonovsky. Int. J. Therm. Sci., 153, 106375 (2020). DOI: 10.1016/j.jithermalsci.2020.106375

[10] A.A. Fedorets, N.E. Aktaev, D.N. Gabyshev, E. Bormashenko, L.A. Dombrovsky, M. Nosonovsky. J. Phys. Chem. C, 123, 23572 (2019). DOI: 10.1021/acs.jpcc.9b08194

[11] A.A. Fedorets, E. Bormashenko, L.A. Dombrovsky, M. Nosonovsky. Philos. T. R. Soc. A, 377, 20190121 (2019). DOI: $10.1098 /$ rsta.2019.0121

[12] L.A. Dombrovsky, A.A. Fedorets, V.Yu. Levashov, A.P. Kryukov, E. Bormashenko, M. Nosonovsky. Atmosphere, 11, 965 (2020). DOI: 10.3390/atmos11090965
[13] W. Suhr. PhyDid B - Didaktik der Physik - Beiträge zur DPG-Frühjahrstagung, Beitrag DD 05.03 (2014).

URL:http://www.phydid.de/index.php/phydid-b/article/view/ 513

[14] L. Jiao, R. Chen, X. Zhu, Q. Liao, H. Wang, L. An, J. Zhu, X. He, H. Feng. J. Phys. Chem. Lett., 10, 1068 (2019). DOI: $10.1021 /$ acs.jpclett.8b03699

[15] V.S. Ajaev, O.A. Kabov. Annu. Rev. Fluid Mech., 53, 203 (2021). DOI: 10.1146/annurev-fluid-030620-094158

[16] А.А. Федорец. Письма в ЖЭТФ, 81 (9), 551 (2005). [A.A. Fedorets. JETP Lett., 81 (9), 437 (2005). DOI: $10.1134 / 1.1984025]$

[17] А.А. Федорец, И.В. Марчук, О.А. Кабов. Письма в ЖТФ, 37 (3), 45 (2011). [A.A. Fedorets, I.V. Marchuk, O.A. Kabov. Tech. Phys. Lett., 37 (2), 116 (2011). DOI: $10.1134 / \mathrm{S} 1063785011020064]$

[18] А.А. Федорец. Письма в ЖТФ, 38 (21), 63 (2012). [A.A. Fedorets. Tech. Phys. Lett., 38 (11), 988 (2012). DOI: $10.1134 / \mathrm{S} 1063785012110077]$

[19] J.-I. Yano. Atmos. Chem. Phys., 14, 7019 (2014). DOI: 10.5194/acp-14-7019-2014

[20] А.А. Федорец. Изв. РАН МЖГ, 43 (6), 97 (2008). [A.A. Fedorets. Fluid Dyn., 43 (6), 923 (2008). DOI: $10.1134 / \mathrm{S} 0015462808060124]$

[21] Э.А. Аринштейн, А.А. Федорец. Письма в ЖЭТФ, 92 (10), 726 (2010). [E.A. Arinshtein, A.A. Fedorets. JETP Lett., 92 (10), 658 (2010). DOI: 10.1134/S0021364010220042]

[22] A.A. Fedorets, L.A. Dombrovsky, P.I. Ryumin. Int. J. Heat Mass Transf., 113, 1054 (2017). DOI: $10.1016 /$ j.ijheatmasstransfer.2017.06.015

[23] A.A. Fedorets, L.A. Dombrovsky. Int. J. Heat Mass Transf., 104, 1268 (2017). DOI: 10.1016/j.ijheatmasstransfer.2016.09.087

[24] A.A. Fedorets, M. Frenkel, E. Bormashenko, M. Nosonovsky. J. Phys. Chem. Lett., 8 (22), 5599 (2017). DOI: $10.1021 /$ acs.jpclett.7b02657

[25] A.A. Fedorets, M. Frenkel, E. Shulzinger, L.A. Dombrovsky, E. Bormashenko, M. Nosonovsky. Sci. Rep., 7, 1888. DOI: $10.1038 / \mathrm{s} 41598-017-02166-5$

[26] O.A. Kabov, D.V. Zaitsev, D.P. Kirichenko, V.S. Ajaev. Nanosc. Microsc. Thermophys. Eng., 21 (3), 60 (2017). DOI: $10.1080 / 15567265.2017 .1279249$

[27] A.A. Fedorets, D.N. Gabyshev, I.V. Marchuk, O.A. Kabov. Interfac. Phenom. Heat Transf., 4 (4), 337 (2020). DOI: $10.1615 /$ InterfacPhenomHeatTransfer.2020037059

[28] A.A. Fedorets, I.V. Marchuk, O.A. Kabov. Interfac. Phenom. Heat Transf., 1 (1), 51 (2013). DOI: $10.1615 /$ InterfacPhenomHeatTransfer.2013007434

[29] А.А. Федорец, И.В. Марчук, О.А. Кабов. Письма в ЖЭТФ, 99 (5), 307 (2014). [A.A. Fedorets, I.V. Marchuk, O.A. Kabov. JETP Lett., 99, 266 (2014). DOI: $10.1134 / \mathrm{S} 0021364014050087]$

[30] А.А. Федорец, И.В. Марчук, П.А. Стрижак, О.А. Кабов. Теплофизика и аэромеханика, 22 (4), 535 (2015). [A.A. Fedorets, I.V. Marchuk, P.A. Strizhak, O.A. Kabov. Thermophys. Aeromech., 22, 515 (2015). DOI: $10.1134 / \mathrm{S} 0869864315040137]$

[31] L. Qiu, S. Dubey, F.H. Choo, F. Duan. Phys. Rev. E, 99, 033106 (2019). DOI: 10.1103/PhysRevE.99.033106

[32] Z. Wu, J. Hao, J. Lu, L. Xu, G. Hu, J.M. Floryan. Phys. Fluids, 32, 012107 (2020). DOI: 10.1063/1.5132350 
[33] M.-J. Liao, L.-Q. Duan. Processes, 9, 142 (2021).

DOI: $10.3390 / p r 9010142$

[34] K. Wang, X. Ma, F. Chen, Z. Lan. Langmuir, 37, 1779 (2021). DOI: 10.1021/acs.langmuir.0c03094

[35] A. Bouillant, T. Mouterde, P. Bourrianne, A. Lagarde, C. Clanet, D. Quéré. Nat. Phys., 14, 1188 (2018). DOI: $10.1038 / \mathrm{s} 41567-018-0275-9$

[36] Л.Г. Лойцянский. Механика жидкости и газа (Гостехиздат, M.-Л., 1950). [L.G. Loitsyanskii. Mechan. Liquids Gases (Pergamon Press, London, 1966). DOI: $10.1016 / C 2013-0-05328-5]$

[37] S. Goldstein. Proc. R. Soc. Lond., 123 (791), 225 (1929). DOI: http://doi.org/10.1098/rspa.1929.0067

[38] Н.Е. Актаев, Т.А. Пенкина. Вестник Тюменского гос. унта. Физико-математическое моделирование. Нефть, газ, энергетика, 6 (1), 166 (2020). [N.E. Aktaev, T.A. Penkina. Tyumen State University Herald. Physical and Mathematical Modeling. Oil, Gas, Energy, 6 (1), 166 (2020). DOI: $10.21684 / 2411-7978-2020-6-1-166-175]$

[39] N.E. Aktaev. Appl. Math. Model., 90, 366 (2021). DOI: $10.1016 /$ j.apm.2020.09.016

[40] O.A. Kabov, D.V. Zaitsev, D.P. Kirichenko, V.S. Ajaev. Interfac. Phenom. Heat Transf., 4, 207 (2016). DOI: 10.1615/InterfacPhenomHeatTransfer.2017020203

[41] D.V. Zaitsev, D.P. Kirichenko, V.S. Ajaev, O.A. Kabov. Phys. Rev. Lett., 119, 094503 (2017). DOI: 10.1103/PhysRevLett.119.094503

[42] P.T. Tsilingiris. Energy Convers. Manag., 49 (5), 1098 (2008). DOI: 10.1016/j.enconman.2007.09.015

[43] Н.С. Шишкин. Облака, осадки и грозовое электричество (Гидрометеоиздат, Л., 1964). [N.S. Shishkin. Clouds, Precipitation, and Thunderstorm Electricity (Gidrometeoizdat, Leningrad, 1964)]

[44] O. Reynolds. Mem. Proc. Manch. Lit. Philos. Soc., 21, 1 (1881).

[45] R. Savino, D. Paterna, M. Lappa. J. Fluid Mech., 479, 307 (2003). DOI: 10.1017/S0022112002003610

[46] M. Geri, B. Keshavarz, G.H. McKinley, J.W.M. Bush. J. Fluid Mech., 833, R3 (2017). DOI: 10.1017/jfm.2017.686

[47] A.A. Fedorets, M. Frenkel, I. Legchenkova, D.V. Shcherbakov, L.A. Dombrovsky, M. Nosonovsky, E. Bormashenko. Langmuir, 35, 15330 (2019).

DOI: 10.1021/acs.langmuir.9b03135

[48] A.V. Shavlov, V.A. Dzhumandzhi, S.N. Romanyuk. Phys. Lett. A, 376 (1), 39 (2011).

DOI: $10.1016 /$ j.physleta.2011.10.032

[49] D.N. Gabyshev. Phys. Wave Phenom., 26 (3), 221 (2018). DOI: $10.3103 / \mathrm{S} 1541308 X 1803007 \mathrm{X}$

[50] С.Н. Андреев, Д.Н. Габышев. Краткие сообщения по физике ФИАН, 45 (9), 3 (2018). [S.N. Andreev, D.N. Gabyshev. Bull. Lebedev Phys. Inst., 45 (9), 257 (2018). DOI: $10.3103 / \mathrm{S} 1068335618090014]$

[51] H. Brenner. Chem. Eng. Sci., 16, 242 (1961). DOI: $10.1016 / 0009-2509(61) 80035-3$

[52] Дж. Хаппель, Г. Бреннер. Гидродинамика при малых числах Рейнольдса (Мир, М., 1976). [J. Happel, H. Brenner. Low Reynolds number hydrodynamics (Springer, Dordrecht, 1983). DOI: 10.1007/978-94-009-8352-6] 